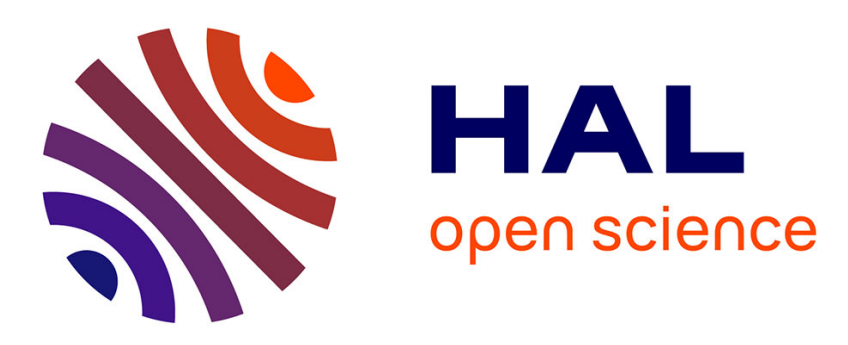

\title{
Use of spherical indentation data changes to materials characterization based on a new multiple cyclic loading protocol
}

Jean-Marc Collin, Gérard Mauvoisin, Philippe Pilvin, Rochdi El Abdi

\section{To cite this version:}

Jean-Marc Collin, Gérard Mauvoisin, Philippe Pilvin, Rochdi El Abdi. Use of spherical indentation data changes to materials characterization based on a new multiple cyclic loading protocol. Materials Science and Engineering: A, 2008, 488, pp.602-622. 10.1016/j.msea.2008.01.041 . hal-01007414

\section{HAL Id: hal-01007414 https://hal.science/hal-01007414}

Submitted on 15 Dec 2018

HAL is a multi-disciplinary open access archive for the deposit and dissemination of scientific research documents, whether they are published or not. The documents may come from teaching and research institutions in France or abroad, or from public or private research centers.
L'archive ouverte pluridisciplinaire HAL, est destinée au dépôt et à la diffusion de documents scientifiques de niveau recherche, publiés ou non, émanant des établissements d'enseignement et de recherche français ou étrangers, des laboratoires publics ou privés. 


\title{
Use of spherical indentation data changes to materials characterization based on a new multiple cyclic loading protocol
}

\author{
Jean-Marc Collin ${ }^{\mathrm{a}, *}$, Gérard Mauvoisin $^{\mathrm{a}}$, Philippe Pilvin ${ }^{\mathrm{b}}$, Rochdi El Abdi ${ }^{\mathrm{a}}$ \\ ${ }^{a}$ LA.R.M.A.U.R., FRE CNRS 2717, Campus de Beaulieu, Université de Rennes 1, 35042 Rennes Cedex, France \\ ${ }^{\mathrm{b}}$ L.G.2M., Université de Bretagne-Sud, Rue de Saint-Maudé, BP 92116, 56321 Lorient Cedex, France
}

\begin{abstract}
An interpretation of spherical indentation experiments and the determination of hardening law parameters is the challenge in this paper. A new protocol based on multiple cyclic loading allowed models describing the indentation data changes to be determined from a numerical study. Inversion of these models, determined for two constitutive equations with isotropic hardening, combined with a sensitivity study enabled the study of the uniqueness of the solution. It is shown that although the new protocol is not necessary to determine the two Hollomon equation parameters, it is necessary to determine accurate values of the three Ludwig equation parameters.

From an experimental study, it was shown that methods based on a "reverse" analysis can be used in order to have an evaluation of the mechanical properties in a first-order approximation.
\end{abstract}

Keywords: Spherical indentation; Materials characterization; Multiple cyclic loading

\section{Introduction}

An instrumented indentation test consists in measuring, simultaneously, the indentation load $P$ and the indentation depth $h$ during the penetration of an indenter into a sample. This test can be used in order to determine some parameters of the work-hardening behavior law of the tested sample. The more commonly used is the Hollomon isotropic work-hardening law defined by the following equation:

$\sigma=\sigma_{\mathrm{y}}^{1-n} E^{n} \varepsilon^{n}$

This relationship gives, in the case of a monotonic solicitation in the elasto-plastic regime, the material flow stress. $E, \sigma_{\mathrm{y}}, n$ and $\varepsilon$, are, respectively the Young modulus, the yield stress, the work-hardening exponent and the strain.

The first identified parameter, from an indentation test, is the reduced Young modulus $E^{*}$ defined by $E^{*}=$ $\left[\left(1-v_{\mathrm{i}}^{2}\right) / E_{\mathrm{i}}+\left(1-v_{\mathrm{s}}^{2}\right) / E_{\mathrm{S}}\right]^{-1}$, where $v$ is the Poisson ratio, $\mathrm{s}$ stands for "sample," whereas i stands for "indenter". The determination of $E^{*}$ is based on the Hertz elastic contacts theory [1].

\footnotetext{
* Corresponding author. Tel.: +33223236282.

E-mail address: jean_marc.collin@yahoo.fr (J.-M. Collin).
}

Sneddon [2] proposed a general solution to the problem of an elastic sample indented by any shape of indenter. In the case of spherical indentation, the contact stiffness is defined as follows:

$S=\frac{\mathrm{d} F}{\mathrm{~d} h}=2 E^{*} a$

where $a$ is the contact radius. When the elastic regime is over, Eq. (2) can still be applied at the beginning of the unloading [3-5]. Following this, Doerner and Nix [6], Loubet et al. [7] and Oliver and Pharr [8] proposed to deduce $E^{*}$ from an indentation test using relationship (2). These methods can be distinguished from one another by the way the contact radius is evaluated. Lastly, Hay and Wolff [9] proposed a correction for the application of the Hertz theory by introducing a factor in order to take into account the radial displacements of material under the indenter.

Concerning the non-elastic behavior, many methods have been proposed to deduce the mechanical parameters, usually deduced from a tensile test, from an indentation test. These methods can be distinguished from one another by the indenter they use (Vickers, sharp, spherical, etc.) and the data they use. Moreover, two kinds of methods can be used to extract mechanical properties from the indentation test. The first is based on the inversion of models established from a numerical study and is called a "reverse" analysis. The second is based on an "inverse" 
analysis. The greater part of established methods are based on a "reverse" analysis, however, Nakamura et al. [10] proposed the determination of the properties of graded materials by using an "inverse" analysis protocol in spherical indentation. We can also quote Bolzon et al. [11] who applied "inverse" analysis to conical indentation. Most of the "reverse" analysis methods are based on the modelling of the representative strain introduced by Tabor [12]. Dao et al. [13] proposed to extract the Eq. (1) parameters from a unique sharp indentation. They also showed that the determined parameters are very sensitive to small variations in the indentation data. This work has been followed by Chollacoop et al. [14] who applied the previous method with two sharp indenters with different apex angles. Then, they showed an improvement in the determination of the material properties with two indenters. Moreover, the use of two sharp indenters significantly decreased the sensitivity of the predicted parameters to the perturbations in the indentation data. Bucaille et al. [15] also proposed using different sharp indenters in order to solve the problem.

The uniqueness of the solution has been fully studied by Cheng and Cheng [16], who demonstrated that in the case of sharp indentation, several sets of Eq. (1) parameters can lead to the same $P(h)$ curve. However, they demonstrated that this problem does not occur in spherical indentation. Indeed, the method of Dao et al. [13] has been extended to the spherical indentation by $\mathrm{Cao}$ and $\mathrm{Lu}$ [17] and led to a unique determination of the Eq. (1) parameters. We can also quote Beghini et al. [18] who determined Eq. (1) parameters from the inversion of a $P(h)$ curve model in spherical indentation.

This paper deals with a study of the uniqueness of the solution in the case of spherical indentation. Moreover, we propose an experimental method based on a "reverse" analysis to evaluate the parameters of two constitutive equations by using a unique indenter and a new multiple cyclic loading protocol.

\section{Data deduced from an indentation test}

Depending on the kind of loading, different data can be deduced from an indentation test (see Fig. 1, the curves of which are deduced from numerical results). If the test consists of a unique loading, the $P(h)$ curve and the total energy $W_{\mathrm{t}}(h)$ changes can be deduced. If the test consists of a loading and an unloading cycle, in addition to $P(h)$ and $W_{\mathrm{t}}(h)$, both the elastic $W_{\mathrm{e}}\left(h_{\max }\right)$ and plastic $W_{\mathrm{p}}\left(h_{\max }\right)$ energies at the end of the loading can be deduced. Moreover, we can deduce the contact stiffness $S\left(h_{\max }\right)$ (defined in Eq. (2)) at the maximal load. Finally, if the test consists of $n$ loading, unloading and reloading cycles, in addition to the previous data we can deduce $n$ points of $W_{\mathrm{e}}\left(h_{(\max ) \mathrm{i}}\right), W_{\mathrm{p}}\left(h_{(\max ) \mathrm{i}}\right)$ and $S\left(h_{(\max ) \mathrm{i}}\right)$ changes. Moreover, from the energies, we can deduce the energy ratio changes as $W_{\mathrm{p}} / W_{\mathrm{t}}\left(h_{(\max ) \mathrm{i}}\right), W_{\mathrm{e}} / W_{\mathrm{t}}\left(h_{(\max ) \mathrm{i}}\right)$ and $W_{\mathrm{e}} / W_{\mathrm{p}}\left(h_{(\max ) \mathrm{i}}\right)$.

Among the three energies, only two are independent. Indeed, they are linked by

$W_{\mathrm{p}}=W_{\mathrm{t}}-W_{\mathrm{e}}$
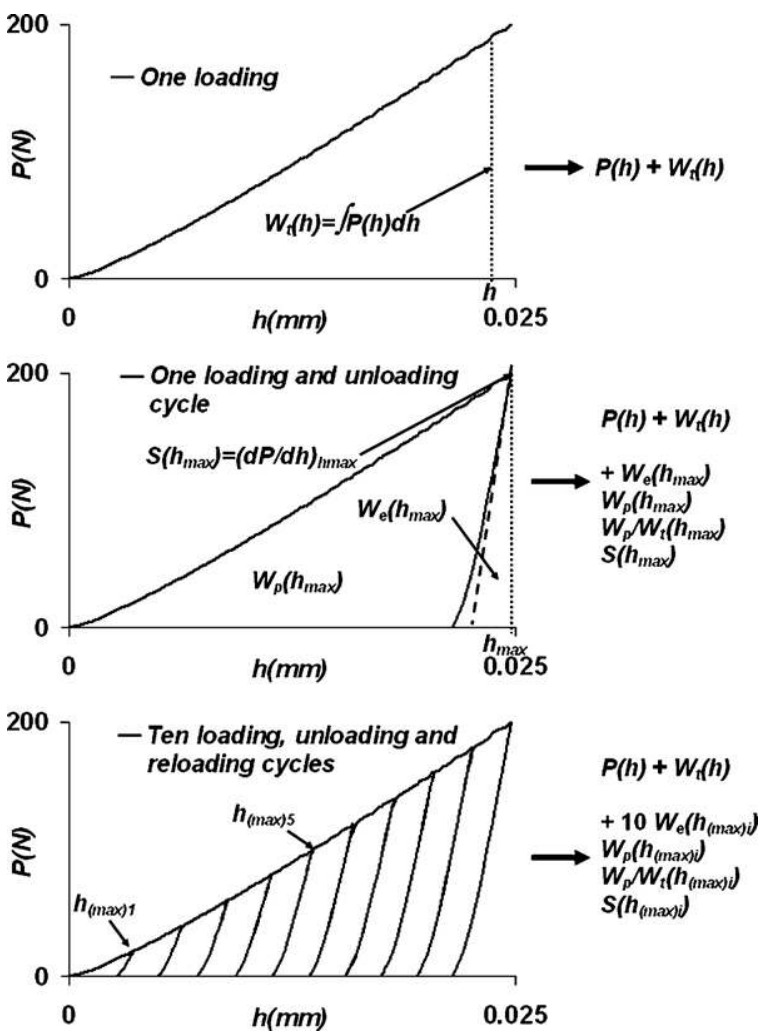

Fig. 1. Different kinds of loading cycles lead to different indentation data.

Moreover, only one energy ratio is sufficient in order to deduce the two others. Indeed, they are linked by

$\frac{W_{\mathrm{p}}}{W_{\mathrm{t}}}=1-\frac{W_{\mathrm{e}}}{W_{\mathrm{t}}}=\left(1+\frac{W_{\mathrm{e}}}{W_{\mathrm{p}}}\right)^{-1}$

This is the reason why, for the three energies, we propose to study both the elastic and total energies. Moreover, although the $W_{\mathrm{p}} / W_{\mathrm{t}}$ ratio is linked to the two studied energies, we propose to study it in order to limit the errors of modelling. Concerning the contact stiffness $S$, because it is obtained by derivating the $P(h)$ curve, it is very sensitive to oscillations of the $P(h)$ curve. For this reason this data is not studied in the following numerical study. Thus, this study concerns the $P(h), W_{\mathrm{e}}, W_{\mathrm{t}}$ and $W_{\mathrm{p}} / W_{\mathrm{t}}$ changes during a spherical indentation test which are deduced from 10 loading, unloading and reloading cycles.

\section{Numerical study}

\subsection{Presentation}

A numerical study was conducted in axi-symetric mode with the finite element (FE) code Cast3M. Fig. 2 shows the FE model where the axi-symetric boundary conditions are imposed on the axi-symetric axis.

The sample is modelled by a bulk divided into several areas. In the contact area, the mesh is made of 8-node elements (named QUA8 in the FE code) with quadratic interpolation. In this area, the size of the elements is less than $4 \mu \mathrm{m}$ in order to obtain $P(h)$ curves with the lowest oscillations. The rest of the sample is 


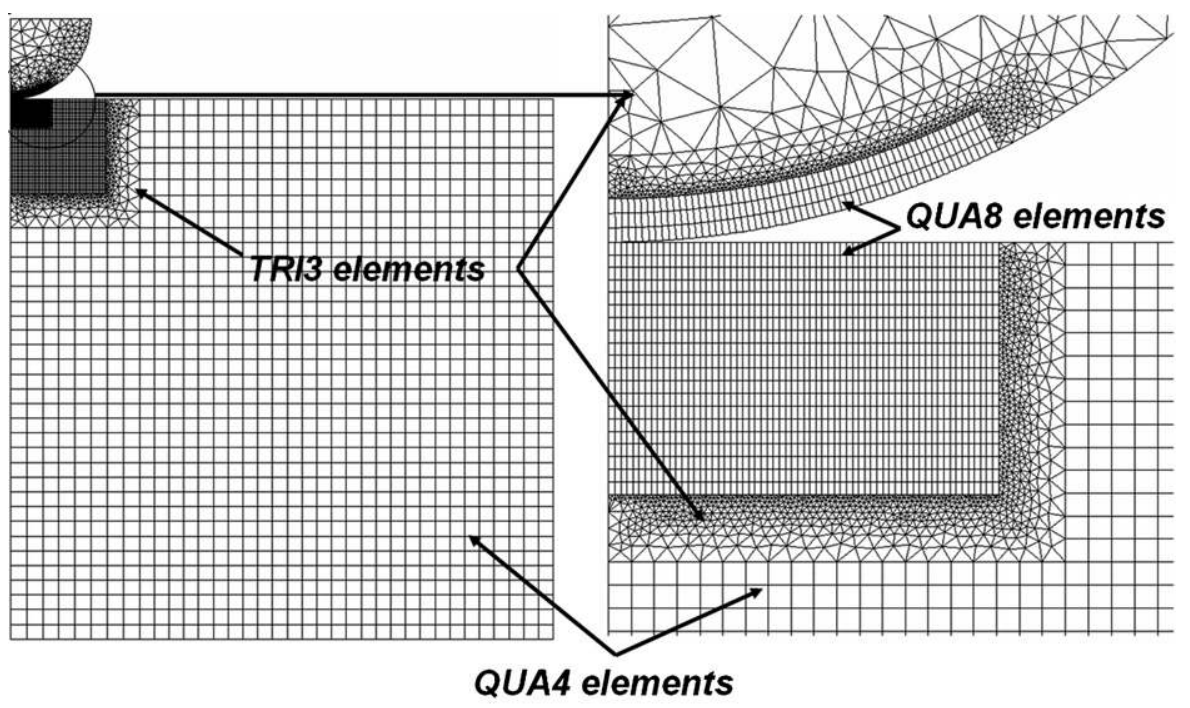

Fig. 2. The FE mesh.

modelled by two areas composed of 4-node elements (named QUA4 in the FE code) with linear interpolation. In the first area, the size of elements is multiplied by 4 and by 80 in the second. Areas with 8-node elements or 4-node elements are separated with transition areas composed of 3-node elements (named TRI3 in the FE code) with linear interpolation.

Concerning the indenter, it is modelled by a quarter of a sphere divided into two areas. In the contact area, as for the sample, the mesh is made of 8-node elements with quadratic interpolation. The rest of the indenter is modelled by an area composed of 3-node elements with linear interpolation.

Because the contact is made between the superior line of the sample and the inferior line of the indenter, the middle nodes of the QUA8 elements are free. In order to solve this problem, two new lines have been created. They are composed of segment elements with linear interpolation. For $n$ QUA8 elements, we have $2 n-1$ nodes in these new lines. Although the friction influence is not negligible, the contact is made without friction between the two new lines in order to focus the study to the plasticity phenomenon.
The sample is described by elasto-plastic behavior with isotropic work hardening. Two constitutive equations have been studied, the Hollomon one (Eq. (1)), and the Ludwig one (Eq. (5)):

$\sigma=\sigma_{\mathrm{y}}+K \varepsilon^{m}$

where $K$ and $m$ describe the work-hardening behavior. It is important to note that Eq. (1) depends on the total strain whereas Eq. (5) depends on the plastic strain only. In this study, the sample's Young modulus is $E_{\mathrm{s}}=210 \mathrm{GPa}$ and the Poisson ratio $v_{\mathrm{s}}=0.3$ (which corresponds to that of steel).

The indenter is described by an elastic behavior with $E_{\mathrm{i}}=600 \mathrm{GPa}$ and $v_{\mathrm{i}}=0.3$ (which corresponds to a tungsten carbide). The indenter radius is fixed to $R=0.5 \mathrm{~mm}$.

In order to follow indentation data changes, 10 loading, unloading and reloading cycles with a maximal load equal to $P_{\max }=200 \mathrm{~N}$ have been programmed. All the nodes of the superior line of the indenter have the same displacement and the load is applied on the first left node of this line. The indenter
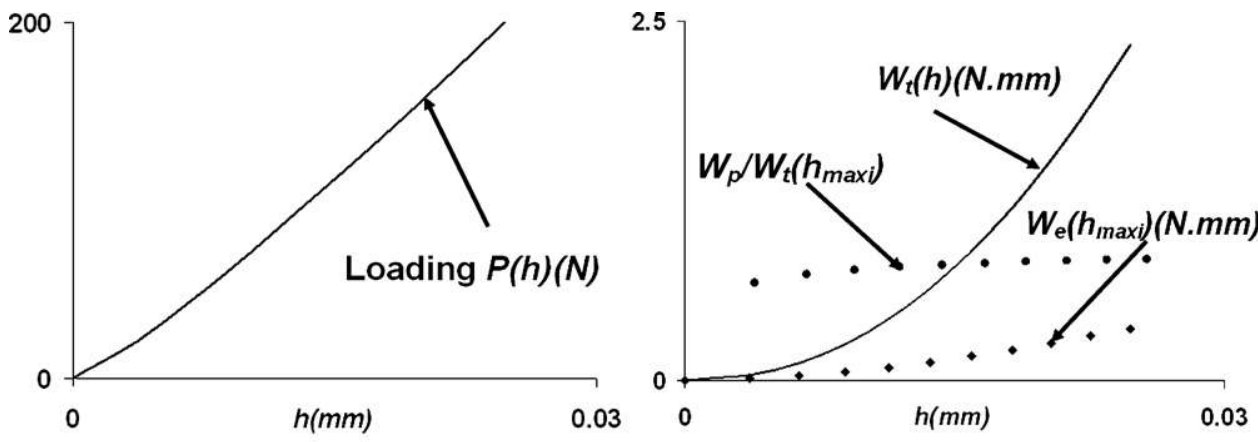

\section{Equation (1) parameters}

$$
\sigma_{y}=450 M P a \text { and } n=0.22
$$

Fig. 3. Typical data deduced from the numerical simulations. 


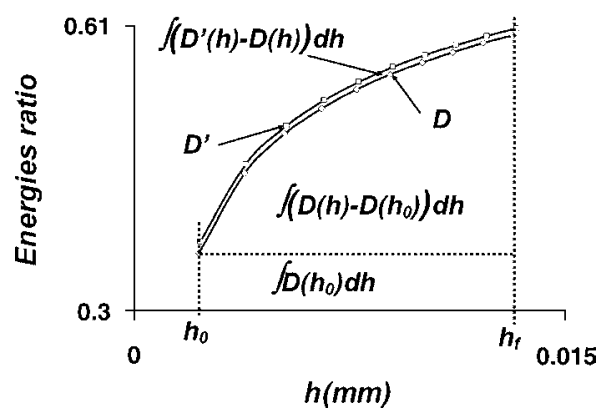

Fig. 4. Quantification of the sensitivity.

moves down into the sample with the bottom of the sample fixed. Figs. 1 and 3 present the typical $P(h)$ curve obtained from the numerical study from which the loading $P(h)$ curve, $W_{\mathrm{e}}, W_{\mathrm{t}}$ and $W_{\mathrm{p}} / W_{\mathrm{t}}$ changes are deduced.

\subsection{Sensitivity study}

The aim of this paragraph is to study the influence of a variation of each behavior law parameter to the indentation data. We can write the sample's behavior law as a function $f$ (Eq. (6)) of $n x_{i}$ behavior law parameters and the strain $\varepsilon$ :

$\sigma=f\left(x_{1}, \ldots, x_{i}, \ldots, x_{n}, \varepsilon\right)$

Thus, indentation data $D$ can be written as a function $g$ (Eq. (7)) of the $n x_{i}$ behavior law parameters and the indentation depth $h$ :

$D=g\left(x_{1}, \ldots, x_{i}, \ldots, x_{n}, h\right)$

If we apply a variation to one parameter $x_{i}$ which becomes $x_{i}^{\prime}$, the indentation data becomes $D^{\prime}$ and can be written as follows:

$D^{\prime}=g\left(x_{1}, \ldots, x_{i}, \ldots, x_{n}, h\right)$

We propose to quantify the sensitivity of indentation data $D$ to the parameter $x_{i}$ (noted $S\left(D / x_{i}\right)$ ) with the following function:

$S\left(D / x_{\mathrm{i}}\right)=\frac{\int_{h_{0}}^{h_{\mathrm{f}}}\left(D^{\prime}(h)-D(h)\right) \mathrm{d} h}{\int_{h_{0}}^{h_{\mathrm{f}}}\left(D(h)-D\left(h_{0}\right)\right) \mathrm{d} h}$

where $h_{0}$ and $h_{\mathrm{f}}$ are presented in Fig. 4. Function (9) has been chosen in order to have an average sensitivity

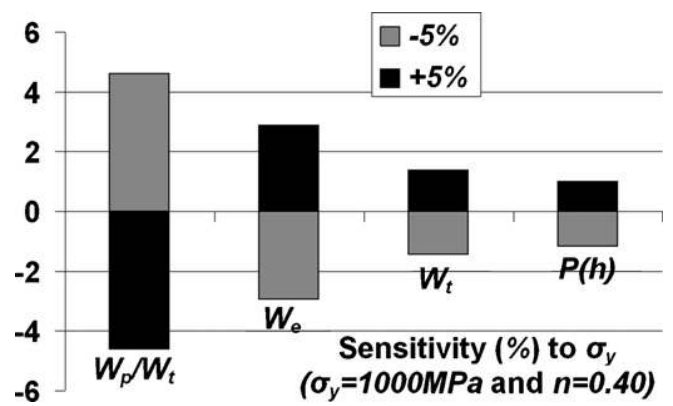

Fig. 5. Symmetry between $+5 \%$ and $-5 \%$ on the sensitivity.

which takes into account the initial value of the indentation data.

In the present work, we have studied the sensitivity of the indentation data to Eq. (5) parameters, i.e. the yield stress $\sigma_{\mathrm{y}}$ and the two work-hardening parameters $K$ and $m$. However, in order to make a graphic representation of the sensitivity changes possible, we distinguish the sample behavior into a yield stress part and a work-hardening part, we then use Eq. (1) notations. Thus, work hardening is represented by the coefficient $n$ of the Eq. (1). In other words, the evolution of the sensitivity of indentation data to $\sigma_{\mathrm{y}}, K$ or $m$ will be presented as a surface which depends on the yield stress and the work hardening quantified by $n$. Twelve sets of parameters have been chosen in order to study the evolution of the sensitivity from a low to a high yield stress $\left(\sigma_{\mathrm{y}}=150 \mathrm{MPa}\right.$ to $\left.\sigma_{\mathrm{y}}=1000 \mathrm{MPa}\right)$ and from a low to high work hardening (quantified by $n=0.02$ to $n=0.4$ ).

For each set of parameters, we have determined $\sigma_{\mathrm{y}}, K$ and $m$ which minimize the difference between Eqs. (5) and (1) in order to have the same flow stress curve. For example, a Hollomon sample with $\sigma_{\mathrm{y}}=150 \mathrm{MPa}$ and $n=0.02$ corresponds to a Ludwig sample with $\sigma_{\mathrm{y}}=150 \mathrm{MPa}, K=24.53 \mathrm{MPa}$ and $m=0.203$. The indentation behavior of these 12 samples has been simulated, and the data deduced from these simulations are considered as the initial ones ( $D$ in Eq. (7)).

In order to verify the symmetry of the sensitivity, a variation of $+5 \%$ and $-5 \%$ has been applied on $\sigma_{\mathrm{y}}, K$ and $m$ for each sample (which leads to 72 simulations). We deduced from these simulations the $D^{\prime}$ data (in Eq. (8)) for a variation $+5 \%$ and

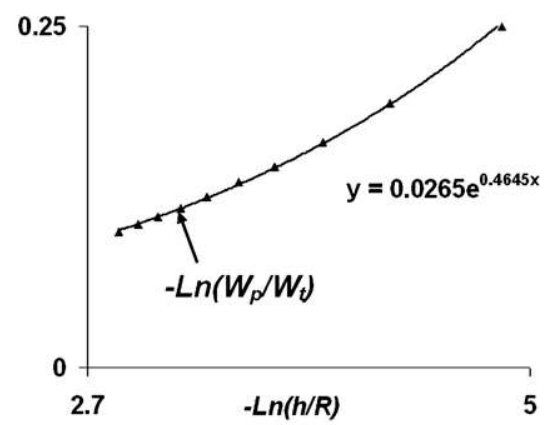

Equation (1) parameters :

$\sigma_{y}=450 \mathrm{MPa}$ and $n=0.15$

Fig. 6. Determination of the functions which describe the indentation data changes. 

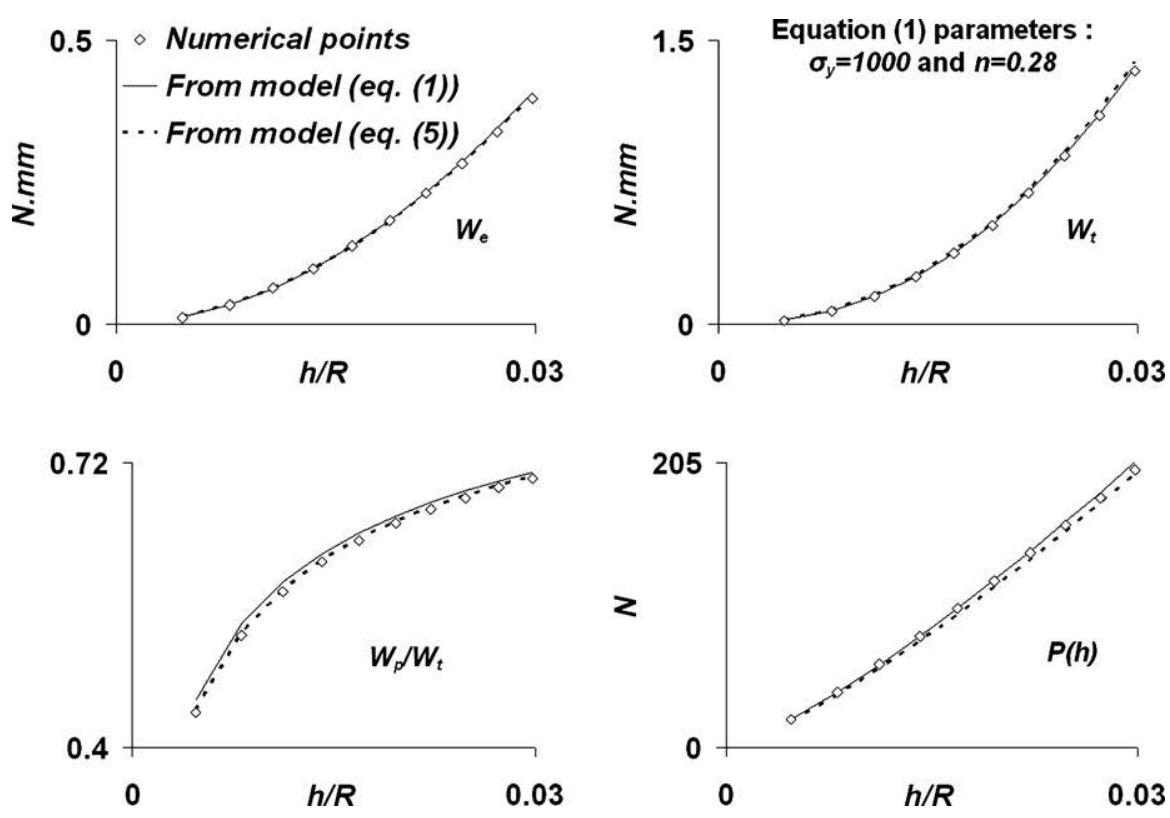

Fig. 7. Comparison between numerical curves and prediction of the models.

$-5 \%$ of the Eq. (5) parameters. We can observe in Fig. 5 that the sensitivity is symmetric data. Thus, we will consider the sensitivity as the average of the absolute values of the sensitivity to $+5 \%$ and $-5 \%$.

The results of the sensitivity study are presented in Fig. A1 (Appendix A). This is composed of four lines representing the sensitivity of the four indentation data $W_{\mathrm{e}}, W_{\mathrm{t}}, W_{\mathrm{p}} / W_{\mathrm{t}}$ and $P(h)$, respectively. The three columns present the sensitivity to the three parameters $\sigma_{\mathrm{y}}, K$ and $m$. For example the first graph in the left top corner shows the sensitivity of the $W_{\mathrm{e}}$ changes to the yield stress in function of the yield stress and the work hardening (quantified by $n$ ). In the following presentation, we discuss the global trend of the sensitivity changes to the yield stress and to the work-hardening parameters.
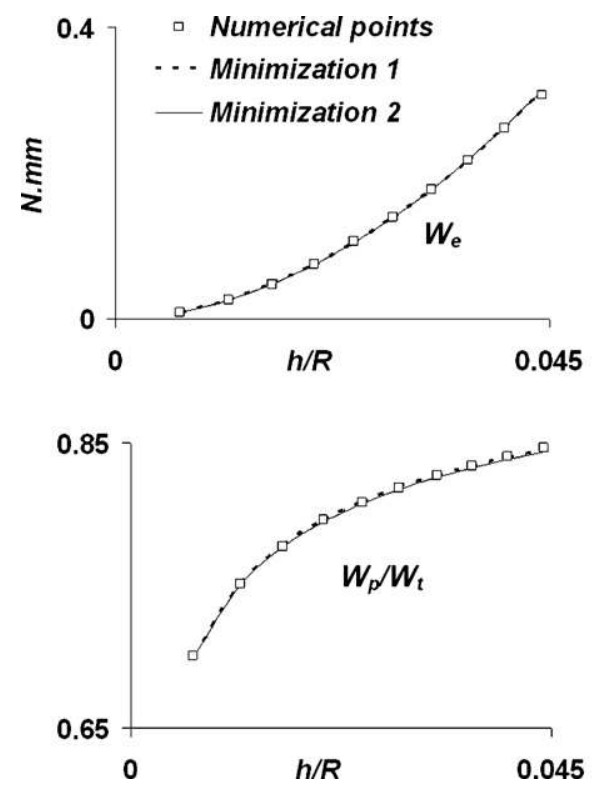

Concerning the evolution of the sensitivity to the yield stress (first column), $W_{\mathrm{e}}, W_{\mathrm{t}}$ and $P(h)$ present the same trend. Moreover, the sensitivities of $W_{\mathrm{t}}$ and $P(h)$ are quite similar. It can be explained by the relationship (10) between $W_{\mathrm{t}}$ and $P(h)$ :

$W_{\mathrm{t}}=\int_{h_{0}}^{h_{\mathrm{f}}} P(h) \mathrm{d} h$

The most important result of the study of the sensitivity to the yield stress is that it increases when the work hardening decreases. We can observe that the work hardening (quantified by $n$ ) has a more important influence than the yield stress on the sensitivity changes except for $W_{\mathrm{p}} / W_{\mathrm{t}}$.

Concerning the evolution of the sensitivity to the workhardening coefficients (second and third columns), we can
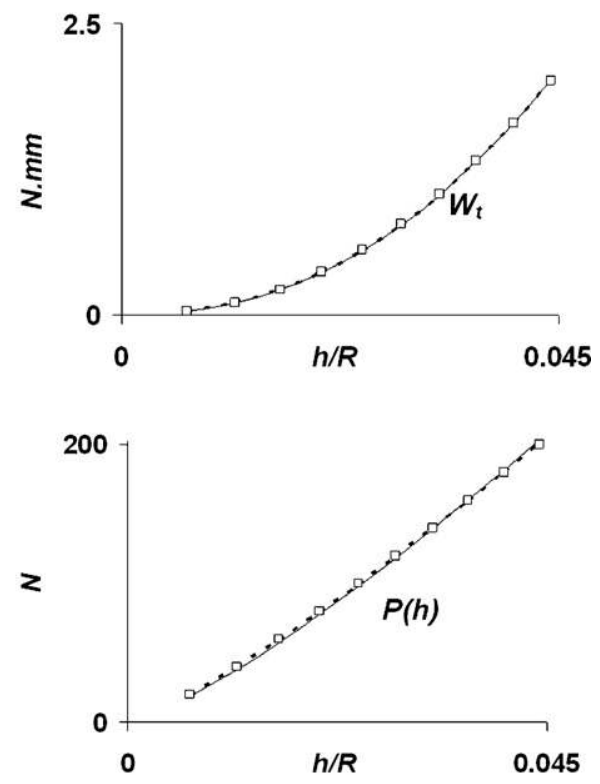

Fig. 8. Several sets of Eq. (5) can lead to the same curve. 


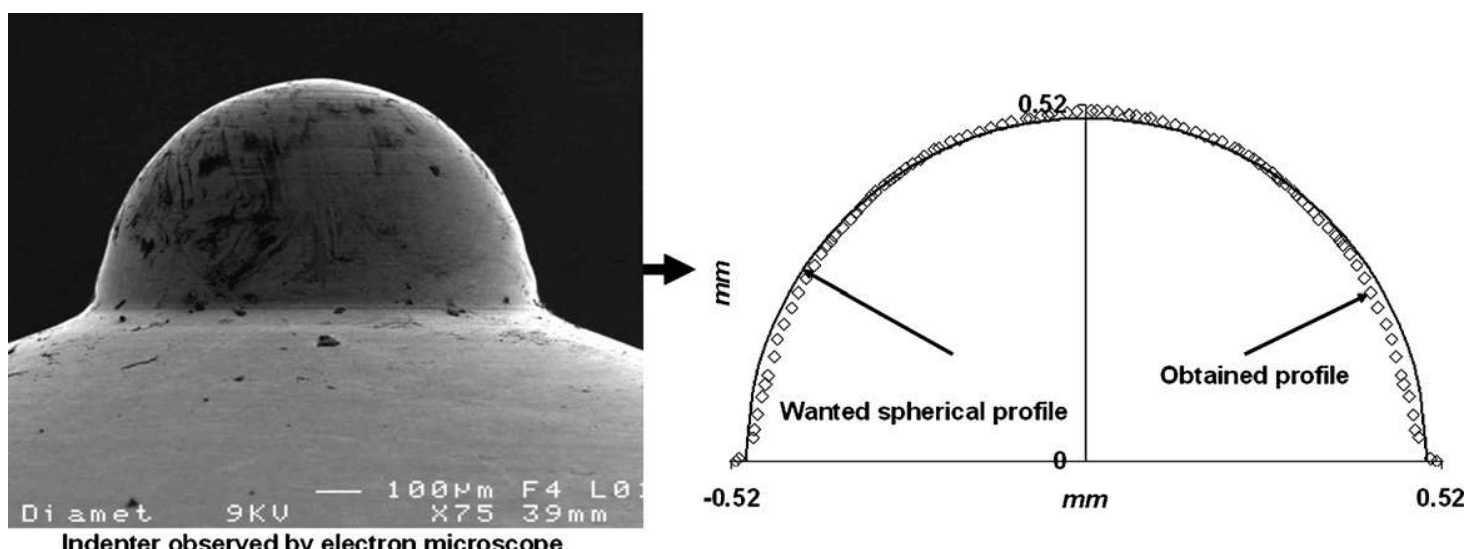

Fig. 9. Our indenter and its profile.

observe that the sensitivity to $K$ and $m$ have the same trend. However, the sensitivity to $m$ is higher than the sensitivity to $K$. The most important result of the study of the sensitivity to the work-hardening coefficients is that no indentation data is sensitive to the work hardening when this work hardening is very low.

We can conclude that the sensitivity can be divided into sensitivity to the yield stress and sensitivity to the work hardening. The maximum value of the sensitivity to the yield stress is compensated by the minimum value of the sensitivity to the work hardening. Moreover, from this study, $W_{\mathrm{e}}$ seems to be the most sensitive data.

\subsection{Determination of models for the four studied indentation data}

The area of the materials studied in the previous paragraph has been extended by considering the large domain of $\sigma_{\mathrm{y}} / E_{\mathrm{s}}$, in order to cover a large area of metallic materials. The yield stress varies between 150 and $3000 \mathrm{MPa}(150,450,720,1000,1400$, 2200 and $3000 \mathrm{MPa}$ ) and the work-hardening exponent of the Eq. (1) varies between 0.02 and $0.4(0.02,0.08,0.15,0.22,0.28$, 0.34 and 0.4 ). The determination of models is then based on 49 materials which have been simulated in order to determine $W_{\mathrm{e}}, W_{\mathrm{t}}, P(h)$ and $W_{\mathrm{p}} / W_{\mathrm{t}}$ changes The parameters of Eq. (5) corresponding to these 49 materials have been determined (as in the previous paragraph) in order to determine models which depend on the parameters of both Eqs. (1) and (5).

Moreover, in order to make the application of the models to different samples and indenters Young modulus and different indenter radii possible, all the data are determined as functions of $\sigma_{\mathrm{y}} / E^{*}, n$ and $h / R$ for Eq. (1) and $\sigma_{\mathrm{y}} / E^{*}, K / E^{*}, m$ and $h / R$ for Eq. (5). In order to have dimensionless data, $W_{\mathrm{e}}$ and $W_{\mathrm{t}}$ are divided by $E^{*} R^{3}$ and $P(h)$ is divided by $E^{*} R^{2}$.

Fig. 6 shows that the evolution of $-\ln \left(W_{\mathrm{e}} / E^{*} R^{3}, W_{\mathrm{t}} / E^{*} R^{3}\right.$ and $\left.P(h) / E^{*} R^{2}\right)$ versus $(-\ln (h / R))$ is well described by an affine function, as for $-\ln \left(W_{\mathrm{p}} / W_{\mathrm{t}}\right)$ versus $(-\ln (h / R))$ which is well described by an exponential function. We can also write that
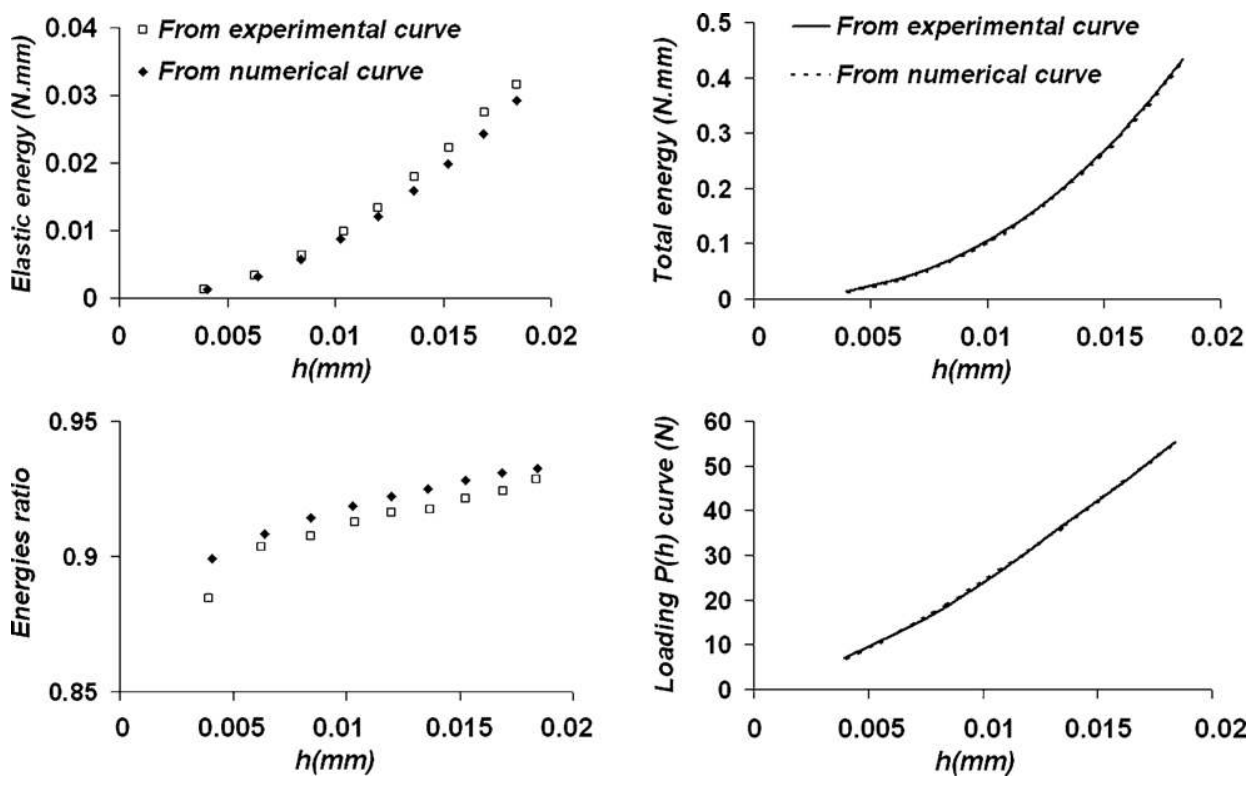

Fig. 10. Comparison between experimental and numerical curves for a $\mathrm{C} 22$ steel. 
Table 1

Results of the experimental characterization for Eq. (1)

\begin{tabular}{|c|c|c|c|c|c|c|c|c|c|c|}
\hline \multirow[t]{2}{*}{ Sample } & \multicolumn{2}{|c|}{ Exact solution } & \multicolumn{2}{|c|}{ Minimization on $W_{\mathrm{e}}$} & \multicolumn{2}{|c|}{ Minimization on $W_{\mathrm{t}}$} & \multicolumn{2}{|c|}{ Minimization on $W_{\mathrm{p}} / W_{\mathrm{t}}$} & \multicolumn{2}{|c|}{ Minimization on $P(h)$} \\
\hline & $\sigma_{\mathrm{y}}(\mathrm{MPa})$ & $n$ & $\sigma_{\mathrm{y}}(\mathrm{MPa})$ & $n$ & $\sigma_{\mathrm{y}}(\mathrm{MPa})$ & $n$ & $\sigma_{\mathrm{y}}(\mathrm{MPa})$ & $n$ & $\sigma_{\mathrm{y}}(\mathrm{MPa})$ & $n$ \\
\hline $\mathrm{C} 22$ & 219 & 0.16 & 182 & 0.21 & 181 & 0.16 & 210 & 0.21 & 174 & 0.19 \\
\hline C100 & 177 & 0.24 & 217 & 0.25 & 163 & 0.22 & 290 & 0.25 & 139 & 0.27 \\
\hline
\end{tabular}

$W_{\mathrm{e}} / E^{*} R^{3}, W_{\mathrm{t}} / E^{*} R^{3}$ and $P(h) / E^{*} R^{2}$ are well described by function $j(11)$ and $W_{\mathrm{p}} / W_{\mathrm{t}}$ by function $k(12)$ :

$j=\left(\frac{h}{R}\right)^{A} \exp (-B)$

$k=\exp \left(-A\left(\frac{h}{R}\right)^{-B}\right)$

where $A$ and $B$ are quantities depending on $\sigma_{y} / E^{*}$ and $n$ in the case of Eq. (1) and on $\sigma_{y} / E^{*}, K / E^{*}$ and $m$ in the case of Eq. (5). From the numerical study, a data base of 49 materials leads to $49 W_{\mathrm{e}}, W_{\mathrm{t}}, P(h)$ and $W_{\mathrm{p}} / W_{\mathrm{t}}$ curves and then to 49 couples $(A, B)$ for each indentation data.

Concerning Hollomon's constitutive Eq. (1), it is possible to represent the evolution of $A$ and $B$ with surfaces which are presented in Fig. B1 (Appendix B). In this figure, functions $A$ and $B$ ( $z$ axis) are determined with the software Table Curve 3D. They depend on $\sigma_{\mathrm{y}} / E^{*}$ ( $x$ axis) and $n$ ( $y$ axis). The proposed functions are the ones which give the best correlation coefficient. The coefficients which come in these functions are given in Tables B1 and B2.

Concerning Ludwig's constitutive equation, it is impossible to represent a surface of the evolution of $A$ and $B$ in function of $\sigma_{\mathrm{y}} / E^{*}, K / E^{*}$ and $m$. These functions have been determined by a minimization with several polynomial functions. If we note $x_{1}=\sigma_{\mathrm{y}} / E^{*}, x_{2}=K / E^{*}$ and $x_{3}=m$, the best found functions are as follows:

$$
\begin{aligned}
A= & a+\sum_{i=1}^{3}\left(a_{i} x_{i}+b_{i} x_{i}^{2}\right)+c_{1} x_{1} x_{2}+c_{2} x_{2} x_{3}+c_{3}\left(x_{1} x_{2}\right)^{2} \\
& +c_{4}\left(x_{2} x_{3}\right)^{2}
\end{aligned}
$$

$B=\left(A+\sum_{i=1}^{3}\left(d_{i} x_{i}^{3}\right)+d_{4} x_{1} x_{3}+d_{5} x_{1} x_{2} x_{3}\right)^{-1}$

The coefficients which come in these functions are given in Tables B3 and B4.

In order to study the accuracy of each model, the comparison has been made between the numerical curves and the prediction of the model for each indentation data (see Fig. 7 for an example). Concerning the models which depend on Eq. (1) parameters, the average accuracy is $2.5 \%, 1.0 \%, 1.6 \%$ and $1.5 \%$, respectively for $W_{\mathrm{e}}, W_{\mathrm{t}}, W_{\mathrm{p}} / W_{\mathrm{t}}$ and $P(h)$. For Eq. (5), the average accuracy becomes, respectively $3.7 \%, 2.6 \%, 1.1 \%$ and $3.4 \%$.

\subsection{Study of the uniqueness of the solution}

The study of the uniqueness of the solution is based on the minimization between the numerical curves and the proposed models for each indentation data and for the two studied constitutive equations. If we note $\sigma_{\text {num }}$ as a point of a numerical curve, $\sigma_{\text {mod }}$ as a point of the curve obtained from a model and $N$ as the number of points, the minimization is commonly made on the following function:

$\sum_{N}\left(\sigma_{\bmod }-\sigma_{\text {num }}\right)^{2}$

Although function (15) gives good results on $W_{\mathrm{p}} / W_{\mathrm{t}}$, it gives very inaccurate results on $W_{\mathrm{e}}, W_{\mathrm{t}}$ and $P(h)$. Indeed, function (15) does not give the same weight to every point of the curve. Then, we suggest minimizing with the following formula:

$\sum_{N}\left(\frac{\sigma_{\text {mod }}-\sigma_{\text {num }}}{\sigma_{\text {num }}}\right)^{2}$

Concerning Hollomon's constitutive equation, formula (16) has been used to inverse the functions (11) and (12) for each indentation data. The results of these inversions, given in Table C1 (Appendix C), show that there is a uniqueness of the solution for each indentation data. We can observe that the lower the work hardening ( $n=0.02$ and 0.08$)$, the lower the precision on the work-hardening exponent $n$. It can be easily explained with the results of the sensitivity study. Indeed, it has been shown that if the work hardening is low, the sensitivity of the four indentation data to the work hardening is close to 0 . If we observe the results of the inversion without considering the lowest values of work-hardening exponent, the average error obtained, respectively on $\sigma_{\mathrm{y}}$ and $n$ is $3.9 \%$ and $4.3 \%$ with $W_{\mathrm{e}}, 2.9 \%$ and $3.4 \%$ with $W_{\mathrm{t}}, 5.0 \%$ and $3.8 \%$ with $W_{\mathrm{p}} / W_{\mathrm{t}}$ and $5.8 \%$ and $6.5 \%$ with $P(h)$. Although the sensitivity study has shown that the most

Table 2

\begin{tabular}{|c|c|c|c|c|c|c|}
\hline \multirow[t]{2}{*}{ Sample } & \multicolumn{3}{|c|}{ Exact solution } & \multicolumn{3}{|c|}{ Results of minimization } \\
\hline & $\sigma_{\mathrm{y}}(\mathrm{MPa})$ & $K(\mathrm{MPa})$ & $m$ & $\sigma_{\mathrm{y}}(\mathrm{MPa})$ & $K(\mathrm{MPa})$ & $m$ \\
\hline $\mathrm{C} 22$ & 219 & 506 & 0.3175 & 243 & 361 & 0.3214 \\
\hline $\mathrm{C} 100$ & 177 & 901 & 0.3789 & 165 & 776 & 0.3618 \\
\hline
\end{tabular}

Results of the experimental characterization for Eq. (5) 
sensitive data is $W_{\mathrm{e}}$, it can be observed that the data which gives the best results is $W_{\mathrm{t}}$. Indeed, this data is less sensitive than $W_{\mathrm{e}}$, but the model of $W_{\mathrm{t}}$ is the most accurate. Moreover, it has been shown that the sensitivity of $W_{\mathrm{t}}$ and $P(h)$ are quite similar. However, the results of the inversion of $W_{\mathrm{t}}$ model are more accurate (accuracy multiplied by two) than the $P(h)$ ones. Indeed, the model of $W_{\mathrm{t}}$ is more accurate than the $P(h)$ one. It is then shown that both the accuracy of model and the sensitivity explain the accuracy of the inversion.

The energy $W_{\mathrm{t}}$ could be calculated by function (10). It would be then not be necessary to program several loading, unloading and reloading cycles in order to determine the $W_{\mathrm{t}}$ changes. One single loading is sufficient to calculate the $W_{\mathrm{t}}$ changes and then to deduce $\sigma_{\mathrm{y}}$ and $n$ from inversion. However, the cyclic loading protocol proposed in this paper is very important in the case of Eq. (5).

Indeed, the minimization between models determined for Eq. (5) and numerical curves for each data does not lead to a unique set of $\sigma_{\mathrm{y}}, K$ and $m$. Fig. 8 and Table $\mathrm{C} 2$ illustrate this fact. Several sets of Eq. (5) parameters lead to the same curve. This shows that one unique indentation data is not sufficient to determine the three parameters of Eq. (5). It is then necessary to minimize (with function (16)) on several indentation data simultaneously. If we consider the four studied data, only two are independent, indeed $W_{\mathrm{t}}$ and $P(h)$ are linked with function (10) and $W_{\mathrm{e}}, W_{\mathrm{t}}$ and $W_{\mathrm{p}} / W_{\mathrm{t}}$ are linked by Eqs. (3) and (4). Although the minimization of both $W_{\mathrm{t}}$ and $W_{\mathrm{e}}$ leads to a unique set of Eq. (5) parameters, for some cases, the results are not very accurate. As an example, for an exact solution $\sigma_{\mathrm{y}}=2200 \mathrm{MPa}, K=6173 \mathrm{MPa}$ and $m=0.46$, the minimization with $W_{\mathrm{e}}$ and $W_{\mathrm{t}}$ simultaneously leads to the solution $\sigma_{y}=2668 \mathrm{MPa}, K=4241 \mathrm{MPa}$ and $m=0.44$ and the minimization with $W_{\mathrm{e}}, W_{\mathrm{t}}$ and $W_{\mathrm{p}} / W_{\mathrm{t}}$ simultaneously leads to the solution $\sigma_{\mathrm{y}}=2340 \mathrm{MPa}, K=6343 \mathrm{MPa}$ and $m=0.47$. This is the reason why we suggest minimizing, simultaneously, on three indentation data. The model of $W_{\mathrm{p}} / W_{\mathrm{t}}$ is more accurate than the $P(h)$ one and this is the reason why the results presented in Table C3 (middle columns) concern the minimization on $W_{\mathrm{e}}$, $W_{\mathrm{t}}$ and $W_{\mathrm{p}} / W_{\mathrm{t}}$ simultaneously. It is then shown that the use of three indentation data leads to a unique set of accurate Eq. (5) parameters. However, on the one hand, it can be observed that the lower the work hardening, the lower the accuracy of $K$. On the other hand very good accuracy is obtained on coefficient $m$ even if the work hardening is low. This can be explained by the form of Eq. (5). In this function, the yield stress $\sigma_{\mathrm{y}}$ and the work hardening (noted $\sigma_{\mathrm{wh}}$ ) are separated. This can be written as

$\sigma_{\mathrm{wh}}=K \varepsilon^{\mathrm{m}}$

If an error $e$ occurs on the work-hardening evaluation, the new work-hardening function becomes

$\sigma_{\mathrm{wh}}^{\prime}=(1+e) K \varepsilon^{\mathrm{m}}=K^{\prime} \varepsilon^{\mathrm{m}^{\prime}}$

Function (18) has to be solved for every $\varepsilon$, and the only one solution is $K^{\prime}=(1+e) K$ and $m^{\prime}=m$. In other words, if an error occurs on the work-hardening evaluation, this error will have an effect exclusively on $K$ and not on $m$. Then, if we calculate the average error without considering the lowest work hardening values, we obtain $8.2 \%, 6.5 \%$ and $2.1 \%$, respectively for $\sigma_{\mathrm{y}}, K$ and $m$.

One can suggest that only the last points of the curves can lead to a unique set of Eq. (5) parameters. This suggestion is true. However, this leads to very inaccurate values of Eq. (5) parameters (see Table C3, last columns).

As a conclusion, although the new cyclic loading protocol is not necessary to determine Eq. (1) parameters, it is necessary to deduce the $W_{\mathrm{e}}$ and $W_{\mathrm{p}} / W_{\mathrm{t}}$ changes and then to deduce accurate values of Eq. (5) parameters.

\section{Experimental study}

\subsection{Presentation}

Two steels were selected (a C100 and a C22) because of their microstructure which leads to good homogeneity. For these samples, a tensile test led to the flow stress-strain curve which was fitted by both Eqs. (1) and (5).

We developed an experimental bench which allows the measurement of the $P(h)$ curve with very good reproducibility. The two important parts of this bench are the indenter and the way the displacement is measured.

Concerning the choice of the indenter, the most common spherical indenters are composed of a sphere which is crimped into the body of the indenter. However, the way the sphere is crimped can induce non-negligible deformations between the body of the indenter and the sphere. This is the reason why we chose to use a monobloc indenter. In this indenter, both the body and the spherical part are machined in the same Tungsten carbide piece. However, despite a spherical part being asked for, it was impossible to obtain a perfectly spherical indenter. Fig. 9 shows an image of the indenter realised by electronic scanning microscope from which the true profile of the indenter was measured. It was then shown that there are non-negligible differences between the true profile and a spherical one. However, this indenter can be considered as an axi-symetric one. In order to compare numerical and experimental curves, the true profile of the indenter was introduced into the FE code as a set of coordinates defining the surface of the indenter.

The displacement was measured by using a capacitive sensor which is fixed near the indenter with an intermediate piece.

Thus our experimental bench allowed us to measure the displacement of the indenter in the sample. However, the needed displacement is the sample surface which is found under the indenter. A previous study [19] proposed a new method to deduce the wanted load-depth curve from the measured one with knowledge of the elastic properties of both the indenter and the sample. This method was based on the determination of the contact radius changes between the indenter and the sample and a modelling of indenter deformations during an indentation test. The contact radius changes were deduced from several loading, unloading and reloading cycles which lead to the determination of the contact stiff- 
ness $S(h)$ changes. Moreover, due to the non-spherical indenter, we proposed an equivalent radius function which depends on the contact radius. The new cyclic loading protocol was then necessary to calculate the equivalent radius changes of our indenter and then to deduce the real sample load-depth curve.

\subsection{Differences between experimental and numerical curves}

In our previous work [19], it is has been shown that experimental and numerical loading curves are quite similar. However, we observed non-negligible differences between experimental and numerical unloading curves. At the end of the unloading, the experimental displacement decreased more quickly than the numerical one. Moreover, the reloading cycles sometimes led to the appearance of hysteresis on the $P(h)$ curve. This phenomenon has been observed and studied by several authors. According to Huber and Tsakmakis [20] the hysteresis is the consequence of a kinematic hardening behavior of the tested material. Moreover, they showed that, in the case of pure kinematic behavior, or both isotropic and kinematic behavior, the displacement decreases more quickly than in the case of pure isotropic behavior during the unloading cycle.

Fig. 10 presents the comparison between the studied data deduced from both the experimental and the predicted numerical curve for C22 steel. It is shown that experimental $P(h)$ and $W_{\mathrm{t}}$ changes are well predicted by numerical simulations. However, we can observe large differences between experimental $W_{\mathrm{e}}$ and $W_{\mathrm{p}} / W_{\mathrm{t}}$ changes and the predicted ones.

\subsection{Experimental characterization of two steels}

Although the differences between experimental and predicted $W_{\mathrm{e}}$ and $W_{\mathrm{p}} / W_{\mathrm{t}}$ changes are non-negligible, we applied function (16) between experiments and models developed for Eq. (1) for each indentation data. The results are presented in Table 1 . They confirm that the data which give the most accurate results is the $W_{\mathrm{t}}$ changes (average accuracy $13 \%$ on $\sigma_{\mathrm{y}}$ and $4 \%$ on $n$ ). The inversion of the $P(h)$ curve gives less accurate results than $W_{\mathrm{t}}$, which can be explained by the accuracy of the corresponding model. Concerning the indentation data which are affected by the unloading, the accuracy of results obtained from the inversion of $W_{\mathrm{e}}$ is near to that obtained for $P(h)$ inversion. This can be explained by the high sensitivity of $W_{\mathrm{e}}$ which compensates the differences between experimental and numerical unloading. Lastly, $W_{\mathrm{p}} / W_{\mathrm{t}}$ gives the least accurate results.

Concerning the determination of the parameters of Eq. (5), the minimization with $W_{\mathrm{e}}, W_{\mathrm{t}}$ and $W_{\mathrm{p}} / W_{\mathrm{t}}$ simultaneously or $W_{\mathrm{e}}, W_{\mathrm{t}}$ and $P(h)$ simultaneously gives very inaccurate results. However, the minimization on $W_{\mathrm{t}}$ changes leads to a unique set of parameters. Although it has been shown in the numerical study that there is no uniqueness if the minimization is made on only one indentation data, we observed uniqueness in this experimental study. This can be explained by the use of a nonspherical indenter with an equivalent radius function. Thus, an indentation test with this kind of indenter can be assimilated to several indentations with different indenter radii. The results of inversion of $W_{\mathrm{t}}$ changes for Eq. (5) are given in Table 2 . The average accuracy is $9 \%$ on $\sigma_{\mathrm{y}}, 21 \%$ on $K$ and $3 \%$ on $m$.

As a conclusion, although some differences occurred between experimental and numerical results, the inversion of models proposed in the numerical study led to an evaluation of the parameters which are in Eqs. (1) and (5). These results also showed that methods based on a "reverse" analysis are limited in accuracy, the more the number of mechanical parameters the less the accuracy of the characterization. This kind of method can be used in order to have an evaluation of the mechanical behavior in a first-order approximation. However, in order to have a more accurate characterization, it will be necessary to use other kinds of methods such as the "inverse" analysis.

\section{Conclusion}

A new kind of indentation protocol is proposed in this paper. It is composed of several loading, unloading and reloading cycles which allow the determination of the indentation data changes. In this study, four indentation data are studied: the elastic and total energies $W_{\mathrm{e}}$ and $W_{\mathrm{t}}$, the plastic to total energy ratio $W_{\mathrm{p}} / W_{\mathrm{t}}$ and the loading $P(h)$ curve. From a numerical study, models describing these data changes have been determined for two constitutive equations with isotropic hardening. These models have allowed us to study the uniqueness of the solution for the two studied constitutive equations. For the first equation with two plasticity parameters, only one indentation data is sufficient to determine the mechanical properties with good accuracy. Moreover, from the numerical results, the best data is the total energy $W_{\mathrm{t}}$ which can be deduced without cyclic loading. However, for the second constitutive equation with three plasticity parameters, it has been shown that several loading, unloading and reloading cycles are necessary to determine accurate values of the mechanical parameters. The numerical study has also shown that both the sensitivity and the accuracy of models explain the accuracy of the results.

In a previous paper, we have shown that the proposed cyclic loading protocol is necessary to deduce the real load-depth curve from the measured one. It is then shown that some differences occur between experimental and numerical unloading curves. These differences justify the low accuracy obtained by inversion of data which depends on the unloading. However, inversion of the total energy which is not sensitive to these problems leads to an evaluation of the parameters of the two studied constitutive equations. In this case, the uniqueness with the second constitutive equation can be explained by the use of an indenter which can be assimilated to several indenters with different radii which are calculated using the new cyclic loading protocol.

It has thus been shown in this paper that in order to evaluate more than two mechanical parameters, it is necessary to increase the experimental data field either by using several indentation data changes or several indenter radii. 
Lastly, this paper shows that methods based on a "reverse" analysis lead to a more or less accurate determination of the mechanical properties. They can, however, give an evaluation of the material's behavior in a first-order approximation and then can be used in order to have a comparison between several materials. The study of other kinds of methods like "inverse" analysis is one of our perspectives in order to increase the accuracy of the characterization.

\section{Acknowledgment}

This research was sponsored by the Brittany Region.

\section{Appendix A. Sensitivity study}

Fig. A1.
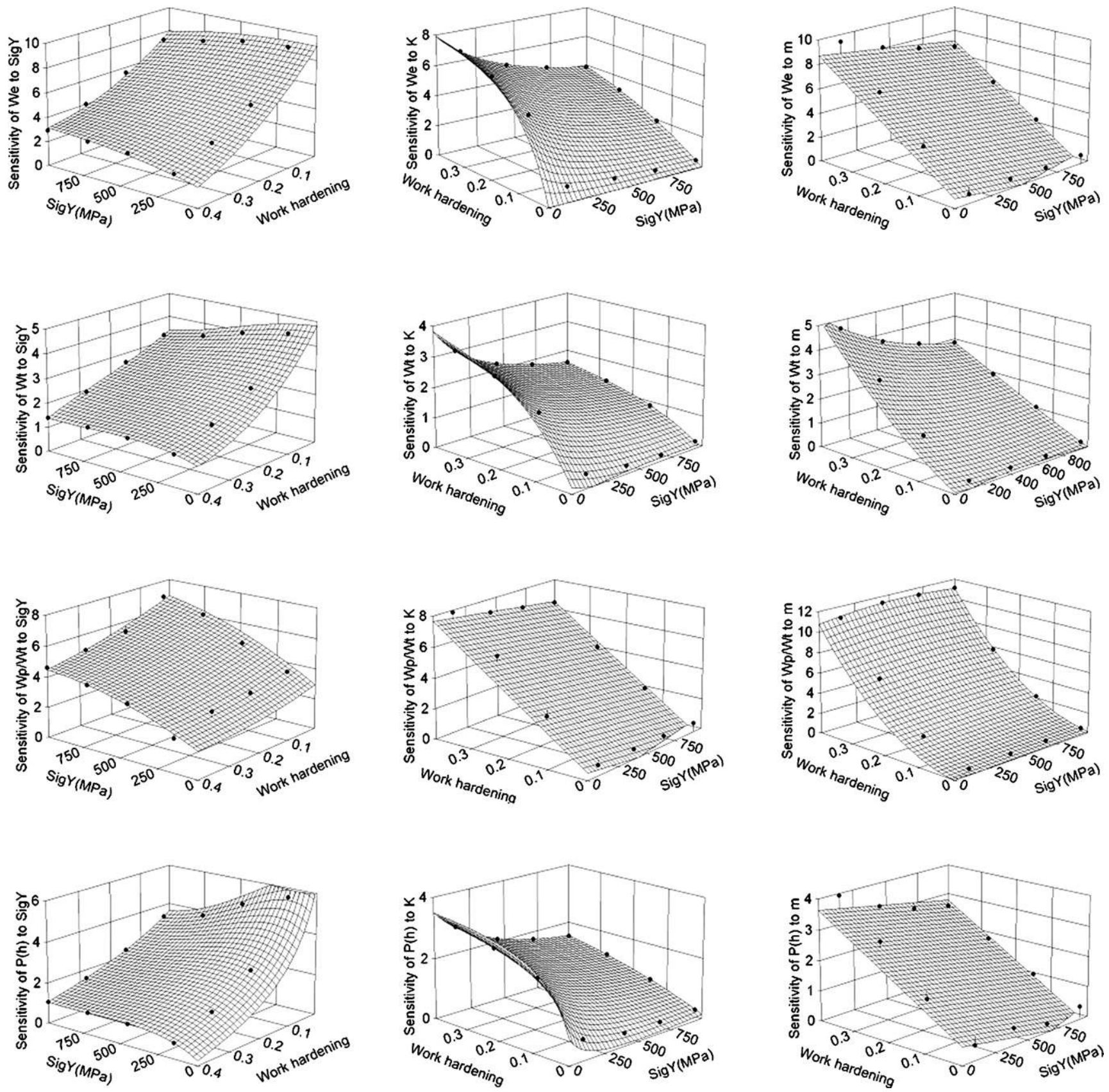

Fig. A1. Sensitivity changes for each studied indentation data. 
Appendix B. Determination of the models

Fig. B1.
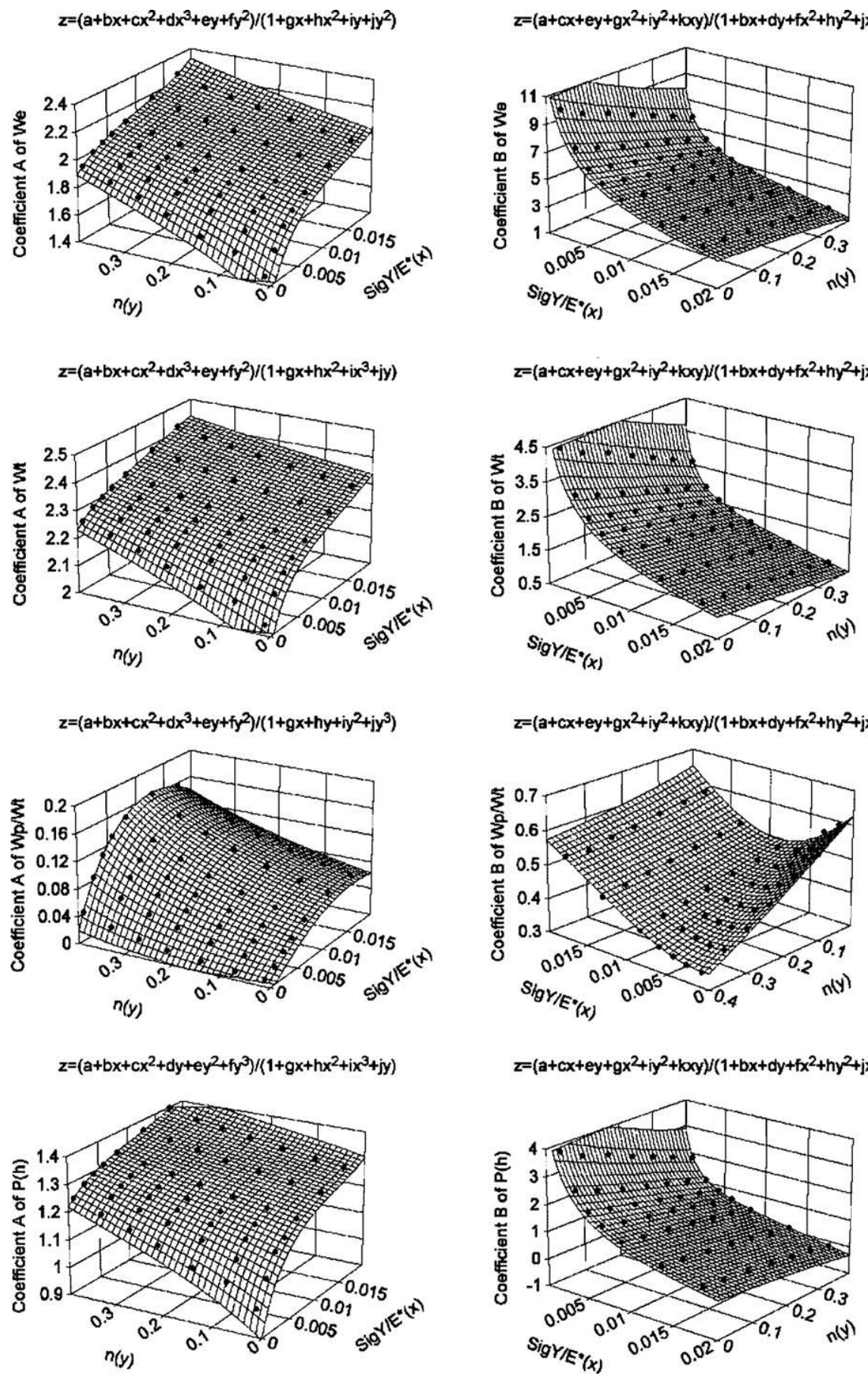

Fig. B1. Functions $A$ and $B$ which come in Eqs. (11) and (12). 
Tables B1-B4.

Table B1

Coefficients which come in function $A$ for Eqs. (11) and (12)

\begin{tabular}{|c|c|c|c|c|c|c|c|c|c|c|}
\hline Data & $a$ & $b$ & $c$ & $d$ & $e$ & $f$ & $g$ & $h$ & $i$ & $j$ \\
\hline$W_{\mathrm{e}}$ & 1.2337 & 368.7702 & -16397.8780 & 44854.0970 & 5.7106 & -2.6740 & 150.2944 & -70.235686 & 2.4492 & -2.0914 \\
\hline$W_{\mathrm{t}}$ & 1.9315 & 526.6240 & -45734.4050 & 1096080.2000 & 5.1262 & 0.7832 & 212.8758 & -19375.6920 & 468759.1300 & 2.1066 \\
\hline$W_{\mathrm{p}} / W_{\mathrm{t}}$ & -0.0075 & 8.2528 & 40.1633 & -9737.7621 & 0.0533 & -0.0566 & 26.1103 & -2.0304 & 0.0756 & 0.8071 \\
\hline$P(h)$ & 0.8946 & 227.6553 & -10699.6670 & 3.6171 & 0.0717 & 1.3472 & 143.5716 & -6922.8572 & -26221.0330 & 2.5028 \\
\hline
\end{tabular}

Table B2

Coefficients which come in function $B$ for Eqs. (11) and (12)

\begin{tabular}{|c|c|c|c|c|c|c|c|c|c|c|c|}
\hline Data & $a$ & $b$ & $c$ & $d$ & $e$ & $f$ & $g$ & $h$ & $i$ & $j$ & $k$ \\
\hline$W_{\mathrm{e}}$ & 12.8151 & 367.3677 & 844.7441 & -0.7832 & -27.8083 & -4794.3394 & -27014.0050 & -1.8007 & 9.0035 & -253.6345 & -578.8095 \\
\hline$W_{\mathrm{t}}$ & 5.7879 & 405.2397 & 392.1503 & -0.6645 & -11.3579 & -4573.9157 & -14009.3360 & -1.8652 & 2.2474 & -215.9234 & -192.3363 \\
\hline$W_{\mathrm{p}} / W_{\mathrm{t}}$ & 0.6262 & -23.8830 & -41.0306 & 0.4967 & -0.4412 & 2001.3517 & 2631.3604 & 0.5303 & -0.0343 & 187.6590 & 137.6183 \\
\hline$P(h)$ & 5.3303 & 309.8288 & 22.8952 & -0.7235 & -12.9210 & -4693.6406 & -7502.3214 & -1.9085 & 6.2890 & -303.3440 & 18.4632 \\
\hline
\end{tabular}

Table B3

Coefficients of function $f_{1}$ (Eq. (13))

\begin{tabular}{|c|c|c|c|c|c|c|c|c|c|c|c|}
\hline Data & $a$ & $a_{1}$ & $a_{2}$ & $a_{3}$ & $b_{1}$ & $b_{2}$ & $b_{3}$ & $c_{1}$ & $c_{2}$ & $c_{3}$ & $c_{4}$ \\
\hline$W_{\mathrm{e}}$ & 0.4799 & 9.5799 & -2.5683 & 5.8946 & -2.8629 & 1.0695 & -6.1122 & -6.9739 & 9.5389 & 0.9933 & -0.0315 \\
\hline$W_{\mathrm{t}}$ & 1.5875 & 6.5688 & -1.2094 & 2.7659 & -3.3348 & -2.6223 & -2.9957 & -7.8405 & 5.1887 & 0.9918 & -1.2836 \\
\hline$W_{\mathrm{p}} / W_{\mathrm{t}}$ & 0.0042 & 8.5243 & 4.2597 & -0.0769 & -256.8118 & -8.2117 & 0.1465 & -71.0339 & -4.6614 & 0.9360 & 35.7140 \\
\hline
\end{tabular}

Table B4

Coefficients of function $f_{2}$ (Eq. (14))

\begin{tabular}{lllllllllllllllll}
\hline Data & $a$ & $a_{1}$ & $a_{2}$ & $a_{3}$ & $b_{1}$ & $b_{2}$ & $b_{3}$ & $c_{1}$ & $c_{2}$ & $c_{3}$ & $c_{4}$ & $d_{1}$ & $d_{2}$ & $d_{3}$ & $d_{4}$ & $d_{5}$ \\
\hline
\end{tabular}

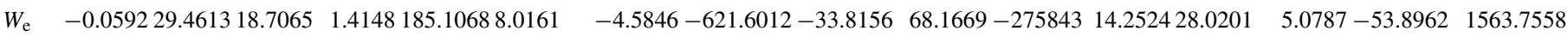

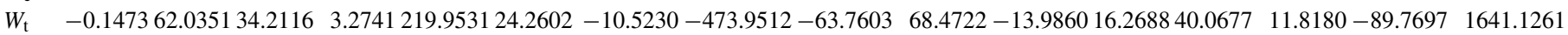
$W_{\mathrm{p}} / W_{\mathrm{t}} \quad 0.478467 .638710 .8059 \quad 6.2035188 .2657-7.8024-3.2472-517.1911-27.8351 \quad 68.4045-25.318715 .405832 .9577 \quad 4.5077-321.29301619 .2977$ $P(h) \quad 0.407256 .1993113 .15177 .5934117 .8745808 .9538-31.7259-78.3279-303.341868 .5109211 .302513 .7692132 .898644 .649445 .6690 \quad 1828.5915$

\section{Appendix C. Study of the uniqueness of the solution}

\section{Tables C1-C3}

Table C1

Results of inversion of models for each indentation data

\begin{tabular}{|c|c|c|c|c|c|c|c|c|c|}
\hline \multicolumn{2}{|c|}{ Exact solution } & \multicolumn{2}{|c|}{ Minimization on $W_{\mathrm{e}}$} & \multicolumn{2}{|c|}{ Minimization en $W_{\mathrm{t}}$} & \multicolumn{2}{|c|}{ Minimization on $W_{\mathrm{p}} / W_{\mathrm{t}}$} & \multicolumn{2}{|c|}{ Minimization on $P(h)$} \\
\hline$\sigma_{\mathrm{y}}(\mathrm{MPa})$ & $n$ & $\sigma_{\mathrm{y}}(\mathrm{MPa})$ & $n$ & $\sigma_{\mathrm{y}}(\mathrm{MPa})$ & $n$ & $\sigma_{\mathrm{y}}(\mathrm{MPa})$ & $n$ & $\sigma_{\mathrm{y}}(\mathrm{MPa})$ & $n$ \\
\hline 150 & 0.02 & 144 & 0.02 & 153 & 0.01 & 204 & 0.03 & 230 & 0.00 \\
\hline 150 & 0.08 & 137 & 0.10 & 124 & 0.11 & 148 & 0.11 & 161 & 0.10 \\
\hline 150 & 0.15 & 172 & 0.13 & 150 & 0.14 & 148 & 0.16 & 165 & 0.13 \\
\hline 150 & 0.22 & 159 & 0.21 & 150 & 0.22 & 134 & 0.23 & 132 & 0.22 \\
\hline 150 & 0.28 & 147 & 0.28 & 154 & 0.28 & 131 & 0.29 & 131 & 0.28 \\
\hline 150 & 0.34 & 136 & 0.34 & 157 & 0.34 & 136 & 0.34 & 139 & 0.34 \\
\hline 150 & 0.4 & 117 & 0.41 & 149 & 0.4 & 127 & 0.41 & 147 & 0.4 \\
\hline 450 & 0.02 & 453 & 0.00 & 487 & 0.00 & 433 & 0.00 & 446 & 0.00 \\
\hline 450 & 0.08 & 471 & 0.07 & 461 & 0.08 & 394 & 0.10 & 411 & 0.09 \\
\hline 450 & 0.15 & 451 & 0.15 & 450 & 0.15 & 393 & 0.17 & 392 & 0.18 \\
\hline 450 & 0.22 & 459 & 0.23 & 455 & 0.22 & 410 & 0.24 & 404 & 0.24 \\
\hline
\end{tabular}


Table C1 (Continued).

\begin{tabular}{|c|c|c|c|c|c|c|c|c|c|}
\hline \multicolumn{2}{|c|}{ Exact solution } & \multicolumn{2}{|c|}{ Minimization on $W_{\mathrm{e}}$} & \multicolumn{2}{|c|}{ Minimization en $W_{\mathrm{t}}$} & \multicolumn{2}{|c|}{ Minimization on $W_{\mathrm{p}} / W_{\mathrm{t}}$} & \multicolumn{2}{|c|}{ Minimization on $P(h)$} \\
\hline$\sigma_{\mathrm{y}}(\mathrm{MPa})$ & $n$ & $\sigma_{\mathrm{y}}(\mathrm{MPa})$ & $n$ & $\sigma_{\mathrm{y}}(\mathrm{MPa})$ & $n$ & $\sigma_{\mathrm{y}}(\mathrm{MPa})$ & $n$ & $\sigma_{\mathrm{y}}(\mathrm{MPa})$ & $n$ \\
\hline 450 & 0.28 & 455 & 0.28 & 456 & 0.28 & 439 & 0.29 & 427 & 0.29 \\
\hline 450 & 0.4 & 454 & 0.40 & 408 & 0.41 & 439 & 0.4 & 451 & 0.40 \\
\hline 720 & 0.02 & 673 & 0.04 & 728 & 0.02 & 561 & 0.08 & 596 & 0.08 \\
\hline 720 & 0.08 & 688 & 0.10 & 707 & 0.09 & 615 & 0.12 & 639 & 0.11 \\
\hline 720 & 0.28 & 720 & 0.28 & 721 & 0.28 & 745 & 0.28 & 724 & 0.28 \\
\hline 720 & 0.34 & 734 & 0.34 & 713 & 0.34 & 759 & 0.33 & 755 & 0.33 \\
\hline 720 & 0.4 & 738 & 0.40 & 648 & 0.41 & 736 & 0.4 & 755 & 0.39 \\
\hline 1000 & 0.02 & 947 & 0.04 & 994 & 0.02 & 877 & 0.06 & 932 & 0.04 \\
\hline 1000 & 0.08 & 935 & 0.10 & 978 & 0.09 & 916 & 0.11 & 952 & 0.10 \\
\hline 1400 & 0.02 & 1410 & 0.02 & 1387 & 0.02 & 1370 & 0.03 & 1395 & 0.02 \\
\hline 1400 & 0.08 & 1386 & 0.08 & 1367 & 0.09 & 1437 & 0.08 & 1462 & 0.07 \\
\hline 1400 & 0.15 & 1400 & 0.15 & 1382 & 0.15 & 1492 & 0.14 & 1519 & 0.12 \\
\hline 1400 & 0.22 & 1416 & 0.21 & 1419 & 0.21 & 1487 & 0.21 & 1534 & 0.19 \\
\hline 1400 & 0.28 & 1425 & 0.27 & 1454 & 0.27 & 1451 & 0.28 & 1505 & 0.26 \\
\hline 1400 & 0.34 & 1442 & 0.33 & 1474 & 0.33 & 1427 & 0.34 & 1494 & 0.32 \\
\hline 1400 & 0.4 & 1450 & 0.39 & 1432 & 0.40 & 1382 & 0.40 & 1440 & 0.39 \\
\hline 2200 & 0.02 & 2350 & 0.00 & 2076 & 0.05 & 2279 & 0.02 & 2205 & 0.02 \\
\hline 2200 & 0.08 & 2254 & 0.07 & 2092 & 0.10 & 2269 & 0.08 & 2240 & 0.07 \\
\hline 2200 & 0.15 & 2192 & 0.15 & 2133 & 0.16 & 2246 & 0.14 & 2232 & 0.14 \\
\hline 2200 & 0.22 & 2155 & 0.23 & 2206 & 0.22 & 2190 & 0.22 & 2155 & 0.23 \\
\hline 3000 & 0.34 & 2927 & 0.34 & 2990 & 0.33 & 2813 & 0.35 & 2972 & 0.33 \\
\hline 3000 & 0.4 & 2809 & 0.44 & 2695 & 0.45 & 3214 & 0.45 & 2954 & 0.41 \\
\hline
\end{tabular}

Table C2

Several sets of parameters can lead to the same curve

\begin{tabular}{|c|c|c|c|c|c|c|c|c|c|}
\hline \multirow{2}{*}{\multicolumn{2}{|c|}{ Exact solution }} & \multicolumn{2}{|c|}{ Minimization on $W_{\mathrm{e}}$} & \multicolumn{2}{|c|}{ Minimization on $W_{\mathrm{t}}$} & \multicolumn{2}{|c|}{ Minimization on $W_{\mathrm{p}} / W_{\mathrm{t}}$} & \multicolumn{2}{|c|}{ Minimization on $P(h)$} \\
\hline & & 1 & 2 & 1 & 2 & 1 & 2 & 1 & 2 \\
\hline$\sigma_{\mathrm{y}}(\mathrm{MPa})$ & 450 & 1253 & 1426 & 1180 & 1058 & 920 & 1398 & 900 & 200 \\
\hline$m$ & 0.39 & 0.36 & 0.36 & 0.35 & 0.36 & 0.39 & 0.41 & 0.34 & 0.49 \\
\hline
\end{tabular}


Table C3

Results of the minimization with $W_{\mathrm{e}}, W_{\mathrm{t}}$ and $W_{\mathrm{p}} / W_{\mathrm{t}}$ for Eq. (5)

\begin{tabular}{|c|c|c|c|c|c|c|c|c|}
\hline \multicolumn{3}{|c|}{ Exact solution } & \multicolumn{3}{|c|}{ Minimization on data changes } & \multicolumn{3}{|c|}{ Minimization on the last points } \\
\hline$\sigma_{\mathrm{y}}(\mathrm{MPa})$ & $K(\mathrm{MPa})$ & $m$ & $\sigma_{\mathrm{y}}(\mathrm{MPa})$ & $K(\mathrm{MPa})$ & $m$ & $\sigma_{\mathrm{y}}(\mathrm{MPa})$ & $K(\mathrm{MPa})$ & $m$ \\
\hline 150 & 25 & 0.2031 & 156 & 14 & 0.2064 & 3 & 368 & 0.3381 \\
\hline 150 & 123 & 0.2336 & 143 & 143 & 0.2455 & 61 & 458 & 0.3331 \\
\hline 150 & 307 & 0.2728 & 148 & 289 & 0.2652 & 77 & 593 & 0.3359 \\
\hline 150 & 609 & 0.3158 & 171 & 553 & 0.3048 & 155 & 621 & 0.3169 \\
\hline 150 & 1,020 & 0.3554 & 184 & 935 & 0.3431 & 190 & 814 & 0.3257 \\
\hline 150 & 1,653 & 0.3973 & 182 & 1,551 & 0.3886 & 80 & 1993 & 0.4241 \\
\hline 150 & 2,628 & 0.4410 & 139 & 2,566 & 0.4390 & 135 & 2560 & 0.4383 \\
\hline 450 & 63 & 0.2487 & 445 & 75 & 0.2522 & 453 & 32 & 0.2481 \\
\hline 450 & 304 & 0.2772 & 359 & 393 & 0.2658 & 485 & 144 & 0.2588 \\
\hline 450 & 720 & 0.3132 & 393 & 724 & 0.3005 & 526 & 214 & 0.2574 \\
\hline 450 & 1,355 & 0.3520 & 444 & 1,187 & 0.3354 & 563 & 360 & 0.2661 \\
\hline 450 & 2,159 & 0.3873 & 505 & 1,727 & 0.3662 & 540 & 1294 & 0.3382 \\
\hline 450 & 3,317 & 0.4244 & 552 & 2,680 & 0.4063 & 645 & 1293 & 0.3324 \\
\hline 450 & 4,990 & 0.4628 & 414 & 4,828 & 0.4579 & 534 & 4076 & 0.4409 \\
\hline 720 & 93 & 0.2737 & 646 & 195 & 0.2753 & 673 & 100 & 0.2680 \\
\hline 720 & 444 & 0.3011 & 647 & 447 & 0.2905 & 702 & 235 & 0.2760 \\
\hline 720 & 1,031 & 0.3353 & 622 & 1,086 & 0.3257 & 741 & 430 & 0.2873 \\
\hline 720 & 1,897 & 0.3719 & 687 & 1,862 & 0.3665 & 791 & 807 & 0.3105 \\
\hline 720 & 2,961 & 0.4051 & 770 & 2,834 & 0.4066 & 785 & 2440 & 0.3903 \\
\hline 720 & 4,451 & 0.4397 & 713 & 4,545 & 0.4465 & 730 & 4362 & 0.4424 \\
\hline 720 & 6,549 & 0.4755 & 778 & 5,529 & 0.4608 & 739 & 4503 & 0.4521 \\
\hline 1000 & 123 & 0.2936 & 802 & 481 & 0.2999 & 858 & 264 & 0.2886 \\
\hline 1000 & 577 & 0.3200 & 818 & 1,093 & 0.3352 & 884 & 533 & 0.3036 \\
\hline 1000 & 1,322 & 0.3529 & 887 & 1,497 & 0.3519 & 903 & 1214 & 0.3379 \\
\hline 1000 & 2,394 & 0.3878 & 1084 & 1,910 & 0.3744 & 868 & 3534 & 0.4262 \\
\hline 1000 & 3,685 & 0.4193 & 1150 & 3,617 & 0.4283 & 843 & 4863 & 0.4500 \\
\hline 1000 & 5,459 & 0.4520 & 1039 & 5,655 & 0.4612 & 879 & 5872 & 0.4601 \\
\hline 1000 & 7,910 & 0.4858 & 1013 & 7,235 & 0.4808 & 887 & 6882 & 0.4638 \\
\hline 1400 & 162 & 0.3164 & 1404 & 304 & 0.3309 & 1092 & 1056 & 0.3413 \\
\hline 1400 & 753 & 0.3416 & 1381 & 973 & 0.3523 & 1100 & 1858 & 0.3712 \\
\hline 1400 & 1,699 & 0.3728 & 1422 & 1,760 & 0.3770 & 1114 & 3146 & 0.4100 \\
\hline 1400 & 3,030 & 0.4059 & 1488 & 2,938 & 0.4089 & 1062 & 5299 & 0.4548 \\
\hline 1400 & 4,600 & 0.4356 & 1572 & 4,416 & 0.4422 & 1001 & 7167 & 0.4755 \\
\hline 1400 & 6,716 & 0.4663 & 1674 & 6,535 & 0.4796 & 931 & 8960 & 0.4843 \\
\hline 1400 & 9,587 & 0.4979 & 1449 & 9,598 & 0.4990 & 3415 & 174 & 0.4010 \\
\hline 2200 & 233 & 0.3510 & 2405 & 214 & 0.3689 & 1488 & 3502 & 0.4173 \\
\hline 2200 & 1,069 & 0.3744 & 2271 & 1,084 & 0.3790 & 2791 & 0 & 0.3823 \\
\hline 2200 & 2,368 & 0.4030 & 2184 & 2,404 & 0.4010 & 2911 & 363 & 0.3885 \\
\hline 2200 & 4,141 & 0.4335 & 2210 & 4,210 & 0.4343 & 2896 & 1185 & 0.3962 \\
\hline 2200 & 6,173 & 0.4605 & 2340 & 6,344 & 0.4717 & 2865 & 2258 & 0.4084 \\
\hline 2200 & 8,847 & 0.4883 & 2391 & 9,481 & 0.5114 & 2823 & 4443 & 0.4399 \\
\hline 2200 & 12,386 & 0.5168 & 2185 & 12,313 & 0.5134 & 3026 & 4718 & 0.4372 \\
\hline 3000 & 298 & 0.3775 & 2985 & 572 & 0.3833 & 2329 & 3226 & 0.4156 \\
\hline 3000 & 1,353 & 0.3997 & 2868 & 1,463 & 0.3914 & 2419 & 3918 & 0.4270 \\
\hline 3000 & 2,962 & 0.4270 & 2764 & 3,046 & 0.4123 & 2615 & 4366 & 0.4334 \\
\hline 3000 & 5,111 & 0.4549 & 2788 & 5,446 & 0.4485 & 2791 & 4643 & 0.4345 \\
\hline 3000 & 7,326 & 0.4720 & 2950 & 8,176 & 0.4870 & 2971 & 4900 & 0.4328 \\
\hline 3000 & 10,674 & 0.5146 & 2832 & 11,619 & 0.5121 & 3111 & 5173 & 0.4322 \\
\hline 3000 & 14,074 & 0.5039 & 2810 & 15,005 & 0.5084 & 3359 & 5787 & 0.4307 \\
\hline
\end{tabular}

\section{References}

[1] H. Hertz, Z. Reine Angew. Math. 92 (1882) 156-171.

[2] I.N. Sneddon, Int. J. Eng. Sci. (1965) 3.

[3] S.I. Bulychev, V.P. Alekhin, Zavod Lab. (1975) 41.

[4] M.Kh. Shorshorov, S.I. Bulychev, V.P. Alekhin, Sov. Phys. Dokl. (1982) 26.

[5] A.P. Ternovskii, V.P. Alekhin, M.Kh. Shorshorhov, M.M. Khurshchov, V.N. Skvortsov, Zavod Lab. 39 (1973) 1973.

[6] M.F. Doerner, W.D. Nix, J. Mater. Res. (1986) 1.
[7] J.L. Loubet, J.M. Georges, G. Meille, Vickers in dentation curves of elastoplastic materials, in: Microindentation Techniques in Materials Science and Engineering, ASTM STP 889, 1986.

[8] W.C. Oliver, G.M. Pharr, J. Mater. Res. 7 (6) (1992) 1564-1583.

[9] J.C. Hay, P.J. Wolff, J. Mater. Res. 16 (5) (2001) 1280-1286.

[10] T. Nakamura, T. Wang, S. Sampath, Acta Mater. 48 (2000) (2000) 4293-4306.

[11] Bolzon, G. Maier, M. Panico, Int. J. Sol. Struct. 41 (2004) (2004) 2957-2975.

[12] D. Tabor, Hardness of Metals, Clarendon Press, Oxford, 1908. 
[13] M. Dao, N. Chollacoop, K.J. Van Vliet, T.A. Venkatseh, S. Suresh, Acta Mater. 49 (2001) 3899-3918.

[14] N. Chollacoop, M. Dao, S. Suresh, Acta Mater. 51 (2003) $3713-$ 3729.

[15] J.L. Bucaille, S. Strauss, E. Felder, J. Michler, Acta Mater. 51 (6) (2003) $1669-1678$.
[16] Y.T. Cheng, C.M. Cheng, Mater. Sci. Eng. R 44 (2004) 91-149.

[17] Y.P. Cao, J. Lu, Acta Mater. 52 (2004) 4023-4032.

[18] M. Beghini, L. Bertini, V. Fontanari, Int. J. Sol. Struct. (2005).

[19] J.-M. Collin, G. Mauvoisin, R. El Abdi, Mech. Mater. 40 (2008) 401-406.

[20] N. Huber, Ch. Tsakmakis, Mech. Mater. 27 (1998) (1997) 241-248. 